\title{
OPTICAL SOA-BASED SWITCHING
}

All-optical switches are fundamental building blocks for high-speed optical networks and basic blocks towards all-optical networks for future communications that utilize Optical Time Division Multiplexing (OTDM) techniques of signal with single channel data rate of hundreds Gbps. It seems that the most promising technology will be based on Semiconductor Optical Amplifiers (SOA). We can arrange these components to some interferometric architectures in which SOAs achieve efficient optical switching rate with low control energy and big optical sampling bandwidth. The interferometric optical switch geometries are presented and characterized in this paper. We discuss their limitation on temporal width of switching window and some of their applications.

\section{Introduction}

In fiber optic communications we can see a continuing growth in transmission capacity and speed basically due to advances in development of optical components and systems in a physical layer (new components are still needed for optical signal processing), for example laser diodes - DFB-lasers, DBR-lasers, Mode-locking lasers...; new hybrid optical amplifiers - Raman/EDFA; and so on. Further, we can see that development in optical components leads up to miniaturization and integration and, therefore, in the future we will prefer those devices witch can satisfy this condition while remaining cost competitive. For future generation of optical networks to utilize the full bandwidth of optical fiber we expect such

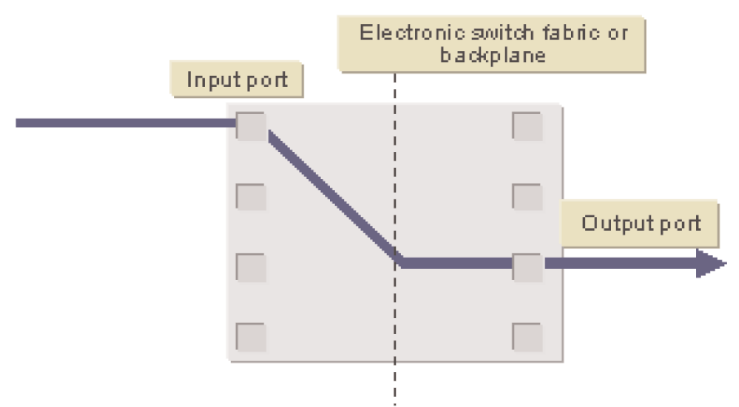

systems (Dense Wavelength Division Multiplexed - DWDM), which will have real bit-rate of individual channels out of range of electronics components. Now practically whole signal processing with optical stream is performed in electrical domain or rarely in hybrid devices - Fig. 1.

In such devices we have to convert an input optical signal to electrical domain where we can perform required processing and then convert it back to an optical signal. The odds are that such devices have their disadvantages (this double conversion is timeconsuming and expensive). Hence we want to overcome or reduce O-E-O conversion. Illustration of such processing can be seen in figure 2 .

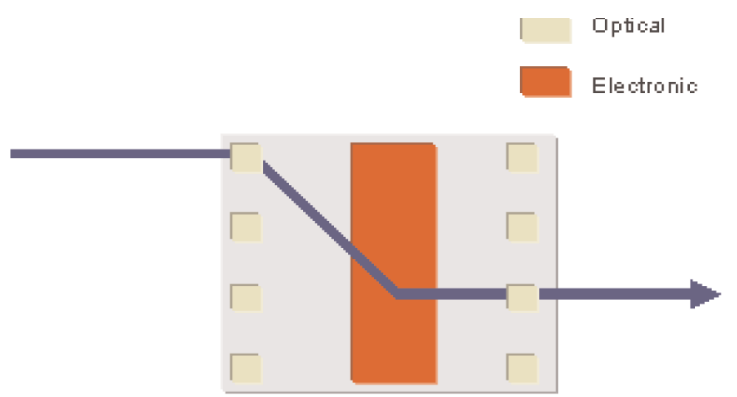

Fig. 1. Illustration of electronic and hybrid optical signal processing

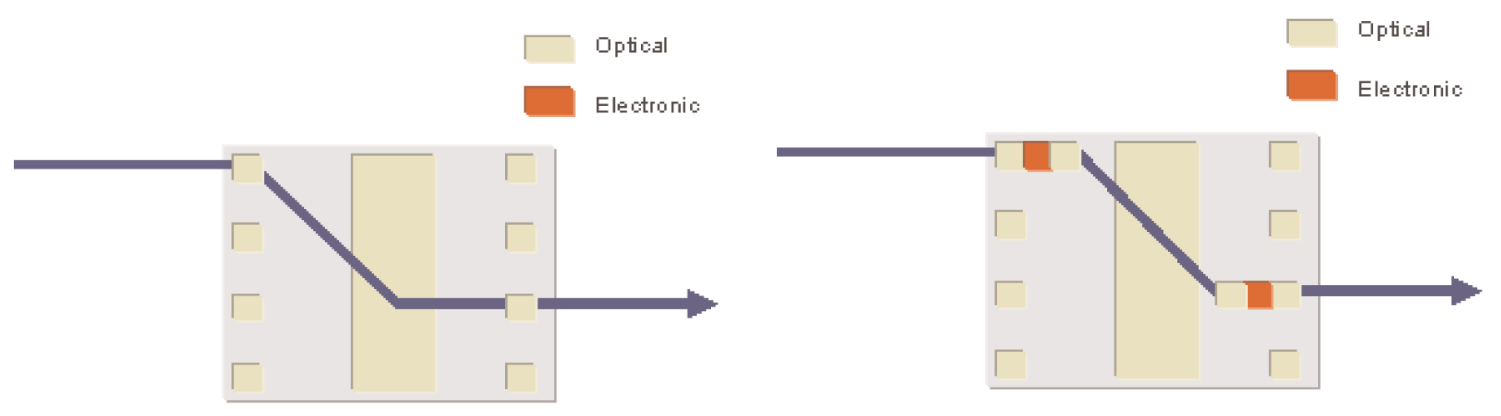

Fig. 2. Illustration of all-optical and hybrid signal processing

\footnotetext{
* Radoslav Odrobiňák, Milan Dado, Róbert Jankovský

Department of telecommunications, University of Zilina, Velký diel, 01026 Žilina, Slovakia, E-mail: odrobinak@fel.utc.sk
} 
In the present time, the developers produce many architectures for all-optical signal processing (but not yet in optimized fabricable application) and SOAs with long recovery times ( $>100 \mathrm{ps})$ seem to demonstrate efficient interferometric all-optical switches to deliver switching and demultiplexing on Tbps data-rate streams. These nonlinearities are typically based on resonance excitation in an actively biased optical amplifier or passive semiconductor nonlinear waveguides. Extensive experimental [1-6] and theoretical [6] analyses have been performed on various configurations of these devices.

\section{SOA architectures}

In this section, we review some of the most promising applications of ultra-fast, SOA-based optical switches under investigation at the University of Zilina. We introduce the architectures for all-optical switches and characterize their performance.

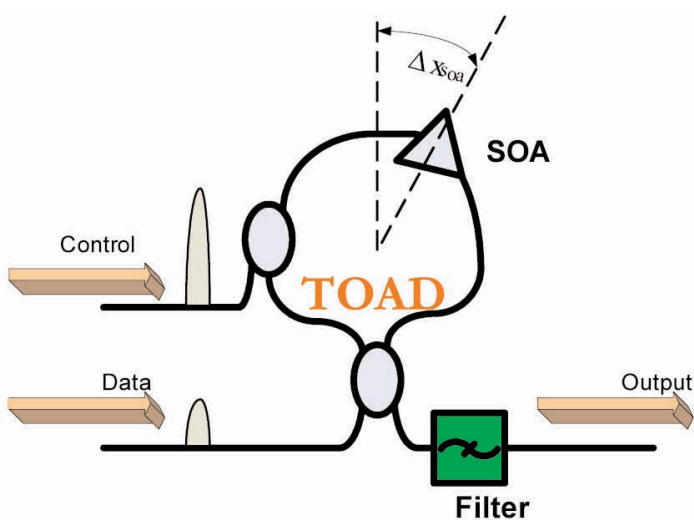

where $c_{\text {fiber }}$ is speed of light in fiber.

Using a similar operating principle it is possible to form other interferometric configuration based on SOA. These architectures improve the integerability and performance of the device, although they require active stabilization if constructed from discrete components. Two variations of the switch in Mach-Zehnder interferometer (MZI) configuration are shown in figure 3. In the absence of the control signals, the MZI is balanced so that the data signals are rejected from the output port. When control pulses are injected into the interferometer, a differential phase shift is briefly introduced between the two arms of the interferometer causing the data pulse to be switched to the output port. The advantage of the Colliding Pulse MZI (CPMZI) is requirement of no output filter. The nominal switching window of CPMZ and SMZ are:

$$
\tau_{\text {win }}=\frac{2 \Delta x_{S O A}}{c_{\text {fiber }}} \quad \tau_{\text {win }}=\Delta t_{c S} \quad \text { respectively. }
$$
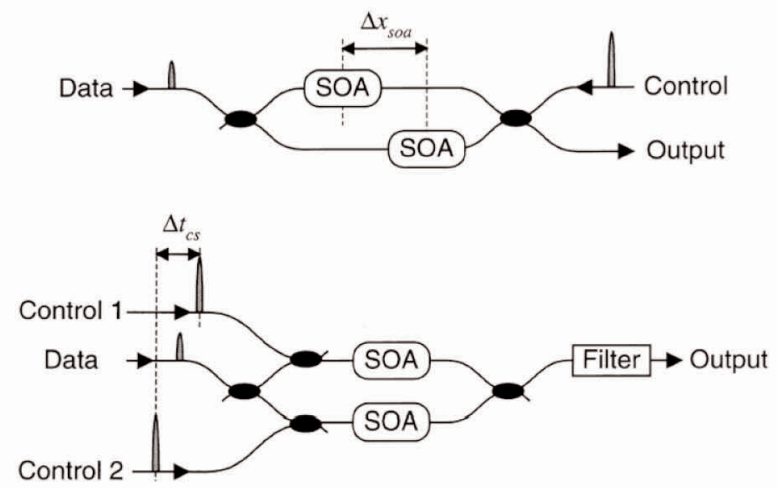

Fig. 3. The main SOA-based architectures: TOAD, CPMZ and SMZ

The main SOA-based architectures are (figure 3): TOAD - Terahertz Optical Asymetric Demultiplexer, CPMZ - Colliding Pulse Mach-Zehnder and SMZ - Symmetric Mach-Zehnder. The TOAD is based on Sagnac interferometer. It was discovered that the temporal width of the switching window could be linearly controlled by adjusting the displacement of the SOA from the midpoint of the loop. Due to dynamics of this configuration, the switching window actually closes earlier than the recovery time of the SOA as the SOA is moved closer to the midpoint. In the absence of a control pulse, data pulses enter the fiber loop, pass through the SOA at different times as they counter-propagate around the loop and recombine interferometrically at the coupler (data is reflected due to same changes in SOA). In the presence of control pulse switching can occur, because control pulse saturate SOA and change its index of refraction, therefore we can achieve different phase shift between two counter-propagating data pulses. The temporal duration of the switching window is determined by the offset of the SOA, $\Delta x_{S O A}$, from the center position of the loop.

$$
\tau_{\text {win }}=\frac{2 \Delta x_{S O A}}{c_{\text {fiber }}}
$$
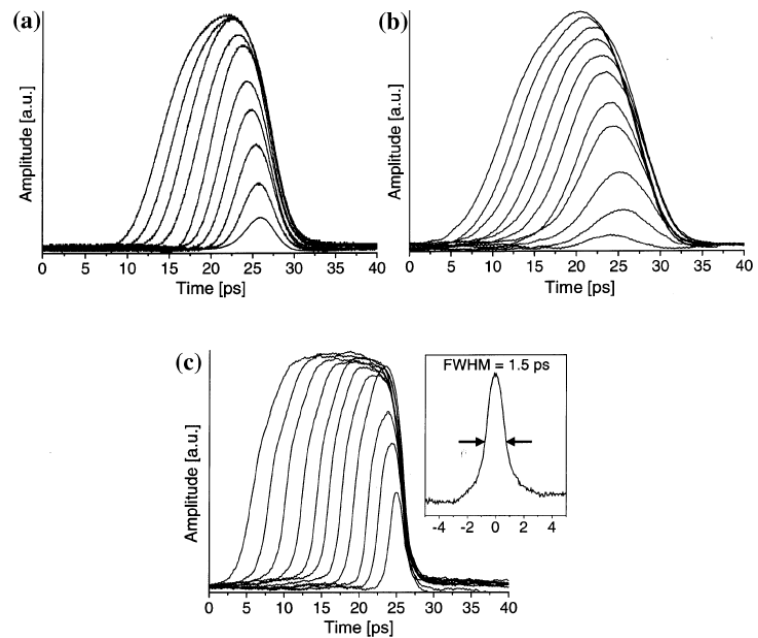

Fig. 4. Optical switching window width simulation results, a) $T O A D, b) C M P Z$ and c) $S M Z$ architecture 


\section{Simulation of the switches}

While many theoretical models have been developed to understand the ultrafast temporal response of SOAs [7-9] we present a summary of the simulated performance of the three geometries introduced in the previous section. The switching windows are acquired by using a scanning pump-probe apparatus described in [6].

The switching window provides information regarding the shape, amplitude, and temporal width of the optical transfer function. This characterization is useful for determining the optical demultiplexing and sampling bandwidth of the switch. For the results described here, various pulse energies, width, and repetition rates were used to demonstrate the effect, where these parameters affect the switching performance.

The shortest switching window achieved by TOAD was about 3.8 ps (FWHM), by CPMZ about 8 ps and by SMZ even 1.5 ps.
This performance indicates that the SMZ structure may be suitable for demultiplexing from $660 \mathrm{Gbps}$ data stream. Reducing the optical switching window further to subpicosecond regimes may be difficult due to SOA gain compression. Further investigation and measurements of these dynamics are important for future research.

\section{Summary}

In this paper we presented new architectures as TOAD, CPMZ and SMZ interferometrical switches. We briefly introduced their performance and operating principle. Using mathematical simulation in VPIprofessionalTool ${ }^{\circledR}$ software we investigated their parameters such as optical temporal window width. We demonstrated that SMZ architecture is ultrafast operating switch able to demultiplex 660 Gbps data stream.

\section{References:}

[1] EISELT, M., PIEPER, W., WEBER, H. G.: "All-optical high speed demultiplexing with a semiconductor laser amplifier in a loop mirror configuration", Electronics Letters 29, str. 1167, 1993.

[2] SOKOLOFF, J. P., PRUCNAL, P. R., GLESK, I., KANE, M.: “A Terahertz Optical Asymmetric Demultiplexer (TOAD)”, IEEE Photon. Technol. Lett. 5: 787-790, 1993

[3] ELLIS, A. D., PATRICK, D. M., FLANNERY, D., MANNING, R. J.: "Ultra-high-speed OTDM networks using semiconductor amplifier-based processing nodes", Journal of Lightwave Technol. 13, 761, 1995

[4] PATEL, N. S., HALL, K. L., RAUSCHENBACH, K. A.: "40-Gbit/s cascadable all-optical logic with an ultrafast nonlinear interferometer", Optics Letters 21, p. 1466, 1996.

[5] NAKAMURA, S., UENO, Y., TAJIMA, K.: "Ultrafast (200-fs switching, 1.5-Tb/s demultiplexing) and high-repetition (10 GHz) operations of a polarization-discriminating symmetric Mach-Zehnder all-optical switch", IEEE Photonics technology Letters 10, 1575, 1998.

[6] TOLIVER, P., RUNSER, R. J., GLESK, I., PRUCNAL, P. R.: “Comparison of Three Nonlinear Interferometric Optical Switch Geometries. Opt. Commun.", vol. 175: 365-373, 2000

[7] HALL, K. L., LENZ, G., DARWISH, A. M., IPPEN, E. P.: "Subpicosecond Gain and Index Nonlinearities in InGaAsP Diode Lasers" Optics Commun. 111, 589-612, 1994.

[8] HONG, M. Y., CHANG, Y. H., DIENES, A., HERITAGE, J. P., DELFYETT, P.J.: "Subpicosecond pulse amplification in semiconductor laser amplifiers: theory and experiment", IEEE Journal of Quantum Electron., 30, p. 1122, 1994

[9] TANG, J. M., SHORE, K. A.: Strong picosecond optical pulse propagation in semiconductor optical amplifiers at transparency, Journal of Quantum Electronics 34, 1263, 1998. 